\title{
Generalized BCS Equations and the Iron-Pnictide Superconductors
}

\author{
Gulshan P. Malik ${ }^{1,2}$, Israel Chávez ${ }^{3}$, Manuel de Llano ${ }^{3}$ \\ ${ }^{1}$ Theory Group, School of Environmental Sciences, Jawaharlal Nehru University, New Delhi, India \\ ${ }^{2}$ B-208 Sushant Lok I, Haryana, India \\ ${ }^{3}$ Instituto de Investigaciones en Materiales, Universidad Nacional Autónoma de México, México DF, México \\ Email: gulshanpmalik@yahoo.com, israelito@ciencias.unam.mx,dellano@unam.mx
}

Received January 6, 2013; revised February 10, 2013; accepted February 20, 2013

Copyright (C) 2013 Gulshan P. Malik et al. This is an open access article distributed under the Creative Commons Attribution License, which permits unrestricted use, distribution, and reproduction in any medium, provided the original work is properly cited.

\begin{abstract}
A detailed quantitative study of the pnictide composite superconductor (CS) $\mathrm{Ba}_{0.6} \mathrm{~K}_{0.4} \mathrm{Fe}_{2} \mathrm{As}_{2}$ is presented in the framework of the recently derived set of generalized BCS equations. Invoking multiple Debye temperatures to take into account anisotropy of the CS, we address the current experimental data on its $T_{c}$ and the (not so clear-cut) gap-values via different theoretical scenarios that attempt to identify the ion species responsible for pairing in it. This is done with the aid of the Bogoliubov's restriction on the BCS dimensionless electron-phonon coupling constant. Significantly, our study sheds light on the gaps which have recently been observed in different iron-pnictide CSs as nodes or line-nodes on the Fermi surface and have evinced considerable interest.
\end{abstract}

Keywords: Generalized BCS Equation; Iron-Pnictide Superconductors; Multiple Gaps

\section{Introduction}

Iron-pnictide superconductors (SCs) [1-4] constitute a promising new family because its members have rather high critical temperatures $\left(T_{c} \mathrm{~s}\right)$; additionally, they readily allow a variety of chemical substitutions to be made. These attributes of the family suggest the possibility in the foreseeable future of fabricating SCs that might meet specific technological needs. It is therefore imperative to develop a theoretical understanding of these materials. To this end, we present in this paper the results of a detailed study, based on the new framework of the generalized BCS equations (GBCSEs) [5], concerned with a prominent member of the family, namely $\mathrm{Ba}_{0.6} \mathrm{~K}_{0.4} \mathrm{Fe}_{2} \mathrm{As}_{2}$ (Ba-As henceforth). We believe that our findings will help in the current flurry in the field.

This paper is organized as follows. In $\$ 2$ we summarize the main experimental features of Ba-As. $\S 3$ recalls the salient features of GBCSEs, which are then applied to this $\mathrm{SC}$ in $\S 4$ by taking into account different scenarios allowed by the current experimental situation. $§ 5$ sums up our findings.

\section{Salient Experimental Features of Ba-As}

The features of Ba-As that concern us here are its $T_{c}$ and the values of its multiple gaps. The experimental situation with regard to the first of these is easily summarized because the values quoted for it in the literature are more or less consistent: $T_{c}=36.5 \mathrm{~K}[3,6] ; 38 \mathrm{~K}[4,6]$ and $37 \mathrm{~K}$ [7]. The situation with regard to the gap-values, however, is not so clear cut.

A broad statement about the $T=0$ gap-values in question was made by [3] on the basis of angle-resolved photoemission spectroscopy (ARPES) measurements reported by [8]. The values so quoted are: 6 and $12 \mathrm{MeV}$. While reporting a gap at about $6 \mathrm{MeV}$ and the absence of a gap at about $12 \mathrm{MeV}$, it was remarked in [6] that this discrepancy (the absence of the larger gap) may be induced by the difference in the methods adopted for determining the gap/s - a remark borne out by what follows. Gaps of single Ba-As crystals were determined both via scanning tunneling microscopy (STM) and spectroscopy in [7] and via penetration-depth measurements in [9]. While the latter of these approaches led to the values of 2.5 and $9.0 \mathrm{MeV}$, the values yielded by the former method are 3.3 and $7.6 \mathrm{MeV}$. The situation is further compounded because: 1) based on specific-heat data, three gap-values have been reported [10] at 3.6, 8.5 and $9.2 \mathrm{MeV}$, whereas 2) the recent ARPES experiment [11] has led to three gaps at 4,7 , and $12 \mathrm{MeV}$ on hole-like 
Fermi surfaces with an additional gap at $9.5 \mathrm{MeV}$ on the electron-like Fermi surface.

For the application of GBCSEs to Ba-As, one requires, first of all, its Debye temperature. Then, in the two-gap scenario, given any two parameters from the set $S=$ $\left\{\Delta_{1}(0), \Delta_{2}(0), T_{c}\right\}$, one is enabled to calculate the remaining parameter. In the scenario in which Ba-As is characterized by two gaps, guided by [7], we adopt for it the set $S=\left\{3.05 \mathrm{MeV}, 8.3 \mathrm{MeV}, T_{c}=38 \mathrm{~K}\right\}$ as our starting point. Also included in this study is an account of our findings in the scenario in which Ba-As may be characterized by three gaps different values for which have been quoted above.

In concluding this summary of the experimental features of Ba-As we note that its Debye temperature is an essential input in this study. This is a parameter rarely quoted for the class of SCs addressed here. The value $274 \mathrm{~K}$ adopted by us is the one quoted in [6]. This will be further discussed below.

\section{Salient Features of GBCSEs}

1) They are based on the premise that superconductivity arises from the formation of Cooper pairs (CPs). CPs in composite superconductors (CSs), however, are regarded as bound via not only one-phonon exchanges, but also via exchanges of two or more species of phonons: these lead to GBCSEs [5]. This implies that CPs in a binary SC, for example, may be bound via two kinds of "glues" or "springs"-leading to two binding energies for the CPs and hence to two gaps.

2) They bypass the issue of the underlying mechanism that brings about pairing. This is so because a two-gap $\mathrm{SC}$ requires the specification of two dimensionless interaction parameters $\lambda_{1}^{c}$ and $\lambda_{2}^{c}$ which occur in three GBCSEs: two for the gaps and one for the $T_{c}$. It is this feature of GBCSEs that enables one to calculate any one of the parameters of the set $S=\left\{\Delta_{1}(0), \Delta_{2}(0), T_{c}\right\}$, given the other two.

3) They take into account the anisotropy of CSs by characterizing them via multiple Debye temperatures (MDTs). These were first introduced by Born and von Karman in connection with a refinement of the Debye theory of specific heat by observing that elastic waves in an anisotropic solid travel with different velocities in different directions. For a review of this and other similar work, we refer to the textbook [12]. In the context of superconductivity, MDTs were resurrected in [13], and subsequently applied $[14,15]$ to a variety of CSs.

\section{GBCSEs Applied to Ba-As}

\subsection{Two-Gap Scenario}

The steps [14] to be followed are:

1) Identify the ion species responsible for pairing in the CS. For Ba-As we assume that these are the $\mathrm{Ba}$ and the $\mathrm{Fe}$ or/and the $\mathrm{Ba}$ and $\mathrm{As}$ ions.

2) Fix the Debye temperatures of the ions identified in 1) via the following equations meant to take into account the anisotropy of the CS:

$$
\begin{gathered}
x \theta_{2}^{c}+(1-x) \theta_{2}^{c}=\theta(x) \\
\frac{\theta_{1}^{c}}{\theta_{2}^{c}}=\left[\frac{1+\sqrt{m_{2} /\left(m_{1}+m_{2}\right)}}{1-\sqrt{m_{2} /\left(m_{1}+m_{2}\right)}}\right]^{1 / 2}
\end{gathered}
$$

These are to be applied to both the $\mathrm{Ba}_{0.6} \mathrm{~K}_{0.4}$ and the $\mathrm{Fe}_{2} \mathrm{As}_{2}$ layers. In (1) $\theta(x)$ is Debye temperature of the $\mathrm{CS}$; in (2) $m_{1}, m_{2}$ are the atomic masses of $A, B$ if the layer is designated as $A_{x} B_{x-1}$. A derivation of (2) is given in [5] where it is assumed that the modes of vibration of the two ions in any layer of the SC are simulated by the modes of vibration of the two bobs of a double pendulum. Note that the ratio of the Debye temperatures in (2) depends on the relative positions of $A$ and $B$ in the double pendulum. In principle, therefore, each ion species may be characterized by two values of the Debye temperature. The values of $\theta_{i}^{c}(i=\mathrm{Ba}, \mathrm{Fe}, \mathrm{As})$ thus obtained appear in Table 1, where they are marked from (1) to (6).

3) To determine the values of $\lambda_{1}^{c}$ and $\lambda_{2}^{c}$, employ the following GBCSEs [5] in which $W_{1}(0)$ and $W_{2}(0)$ are to be identified with $\Delta_{1}(0)$ and $\Delta_{2}(0)[14,15]$, respectively

$$
\begin{gathered}
1=\lambda_{1}^{c} \ln \left[1+\frac{2 \hbar\left(\omega_{D}\right)_{1}^{c}}{\left|W_{1}(0)\right|}\right] \\
1=\lambda_{1}^{c} \int_{0}^{\theta_{1}^{c} / 2 T_{c}} \frac{\tanh (x)}{x} \mathrm{~d} x+\lambda_{2}^{c} \int_{0}^{\theta_{2}^{c} / 2 T_{c}} \frac{\tanh (x)}{x} \mathrm{~d} x \\
1=\lambda_{1}^{c} \ln \left[1+\frac{2 \hbar\left(\omega_{D}\right)_{1}^{c}}{\left|W_{2}(0)\right|}\right] \\
+\lambda_{2}^{c} \ln \left[1+\frac{2 \hbar\left(\omega_{D}\right)_{2}^{c}}{\left|W_{2}(0)\right|}\right],\left(\hbar \omega_{D}=k_{B} \theta\right)
\end{gathered}
$$

where $k_{B}$ is the Boltzmann constant. The $\lambda$ s can be determined with the input of: a) $\Delta_{1}(0)$ and $\Delta_{2}(0)$ into (3) and (5); b) $\Delta_{1}(0)$ and $T_{c}$ into (3) and (4); c) $T_{c}$ and $\Delta_{2}(0)$ into (4) and (5). One is then enabled to calculate $T_{c}$ via (4), or $\Delta_{2}(0)$ via (5), or $\Delta_{1}(0)$ via (3).

4) Regardless of how $\lambda_{1,2}^{c}$ are determined, impose on them the Bogoliubov upper bound on $\lambda$ [16] that each dimensionless coupling constant be positive definite and satisfy

$$
\lambda_{1,2}^{c}<0.5
$$

As will be seen, this criterion is an invaluable guide in this study.

5) If one follows either approach a) or b) of paragraph 
Table 1. In the first three columns are given the Debye temperatures $(\theta)$ of Ba, Fe and As ions obtained via Equations (1) and (2) with $\theta(x)=274 \mathrm{~K}$ [6]. For each pair of Debye temperatures given in column (4), $\lambda$ s given in column (6) are calculated with the initial input of $W_{2}=8.3 \mathrm{MeV}$ and $T_{c}=38 \mathrm{~K}$. Since these $\lambda$-values (e.g., 28.61, -25.87 against the pair of Debye temperature $(1,3)$ ) in each case violate the constraint given in Equation (6), we first vary $W_{2}(0)$ (retaining $\left.T_{c}=38 \mathrm{~K}\right)$ till $\lambda$-values that satisfy constraint (6) are obtained. This procedure is repeated by varying $T_{c}$ (retaining $\left.W_{2}(0)=8.3 \mathrm{MeV}\right)$. Each pair of such acceptable $\lambda$-values leads via Equation (3) to two possible values for the smaller gap, one of which $\left(\sim 10^{-2} \mathrm{MeV}\right)$ seems to be a signature of the node or line-node reported in [17-19].

\begin{tabular}{|c|c|c|c|c|c|c|c|c|c|c|}
\hline \multicolumn{3}{|c|}{ Debye Temperatures [K] } & \multicolumn{4}{|c|}{$\mathrm{Ba}+\mathrm{Fe}$ scenario } & \multicolumn{4}{|c|}{$\mathrm{Ba}+$ As scenario } \\
\hline$\theta_{\mathrm{Ba}}[\mathrm{K}]$ & $\theta_{\mathrm{Fe}}[\mathrm{K}]$ & $\theta_{\mathrm{As}}[\mathrm{K}]$ & $\begin{array}{c}\text { Debye } \\
\text { temps used }\end{array}$ & $\begin{array}{c}W_{2}, T_{c} \\
{[\mathrm{MeV}, \mathrm{K}]}\end{array}$ & $\lambda_{\mathrm{Ba}}, \lambda_{\mathrm{Fe}}$ & $\begin{array}{c}W_{1(\mathrm{Ba})}, W_{1(\mathrm{Fe})} \\
{[\mathrm{MeV}]}\end{array}$ & $\begin{array}{c}\text { Debye } \\
\text { temps used }\end{array}$ & $\begin{array}{c}W_{2}, T_{c} \\
{[\mathrm{MeV}, \mathrm{K}]}\end{array}$ & $\lambda_{\mathrm{Ba}}, \lambda_{\mathrm{As}}$ & $\begin{array}{c}W_{1(\mathrm{Ba})}, W_{1(\mathrm{As})} \\
{[\mathrm{MeV}]}\end{array}$ \\
\hline & & & & $8.3,38$ & $28.61,-25.87$ &,-- & & $8.3,38$ & $34.16,-31.74$ &,-- \\
\hline 326.21 & 399.43 & 375.92 & $(1,3)$ & $6.4,38$ & $0.311,0.118$ & $2.35,1.40 \times 10^{-2}$ & $(1,5)$ & $6.4,38$ & $0.265,0.164$ & $1.33,0.144$ \\
\hline \multirow[t]{2}{*}{ (1) } & (3) & (5) & & $8.3,48$ & $0.303,0.169$ & $2.15,0.192$ & & $8.3,48$ & $0.236,0.236$ & $0.835,0.958$ \\
\hline & & & & $8.3,38$ & $-1.245,2.337$ &,-- & & $8.3,38$ & $-0.684,1.704$ &,-- \\
\hline 124.58 & 172.08 & 148.57 & $(1,4)$ & $7.1,38$ & $0.028,0.571$ & $1.25 \times 10^{-14}, 6.23$ & $(1,6)$ & $7.1,38$ & $0.151,0.439$ & $7.49 \times 10^{-2}, 2.92$ \\
\hline \multirow[t]{7}{*}{ (2) } & (4) & (6) & & $8.3,44$ & $0.136,0.474$ & $3.60 \times 10^{-2}, 4.10$ & & $8.3,44$ & $0.232,0.372$ & $0.766,1.87$ \\
\hline & & & & $8.3,38$ & $1.257,-0.271$ &,-- & & $8.3,38$ & $1.259,-0.279$ &,-- \\
\hline & & & $(2,3)$ & $7.5,38$ & $0.480,0.146$ & $3.05,7.31 \times 10^{-2}$ & $(2,5)$ & $7.3,38$ & $0.476,0.151$ & $2.99,8.63 \times 10^{-2}$ \\
\hline & & & & $8.3,43$ & $0.429,0.203$ & $2.31,0.497$ & & $8.3,43$ & $0.423,0.211$ & $2.23,0.572$ \\
\hline & & & & $8.3,38$ & $1.679,-0.753$ &,-- & & $8.3,38$ & $2.28,-1.35$ &,-- \\
\hline & & & $(2,4)$ & $7.5,38$ & $0.460,0.236$ & $2.76,0.436$ & $(2,6)$ & $7.6,38$ & $0.494,0.228$ & $3.26,0.327$ \\
\hline & & & & $8.3,42$ & $0.259,0.439$ & $0.469,3.41$ & & $8.3,41$ & $0.390,0.356$ & $1.79,1.64$ \\
\hline
\end{tabular}

Notes on tables: Atomic masses used in the calculation of Debye temperatures are: $137.33 \mathrm{~K}(\mathrm{Ba}), 39.098 \mathrm{~K}(\mathrm{~K}), 74.92 \mathrm{~K}(\mathrm{As}), 55.847 \mathrm{~K}(\mathrm{Fe}) .{ }^{*} \mathrm{Entry}$ marked (1) is obtained when $\mathrm{Ba}$ is the upper bob (and $\mathrm{K}$ the lower one; $\theta_{K}^{c}$ are not given as they are not used as shown) in the double pendulum; the one marked (2) is obtained with bobs interchanged. ${ }^{*}$ The $\theta_{i}^{c}$ in next two columns are to be similarly interpreted. ${ }^{*}$ Note, however, that a pair of $\theta_{i}^{c} \mathrm{~s}$ that satisfies Equation (1) is (3) and (6), not (3) and (5).

3), then, while invoking (3), a guess is required about the ion species $(\mathrm{Ba}$, or $\mathrm{Fe}$, or $\mathrm{As})$ that is responsible for $W_{1}(0)$. This issue is circumvented in approach c), which is also the approach one must follow if $\Delta_{1}(0)$ is the parameter known with the least accuracy-as is generally the case. Therefore, we first give below an account of approach c).

6) Let the $\mathrm{Ba}$ and $\mathrm{Fe}$ ion species be invoked to determine the two $\lambda \mathrm{s}$ via (4) and (5). Note that one cannot assume that $\theta_{\mathrm{Fe}}^{c}=\theta_{\mathrm{As}}^{c}=\theta(x)$ because it causes the characteristic determinant of the equations to vanish whence the $\lambda$ 's become indeterminate. The combinations of $\theta$ s (Table 1) that we need to consider are: $(1,3),(1,4),(2,3)$ and $(2,4)$. Further, we require as input the values of $T_{c}$ and $W_{2}(0)$; guided by [7], we adopt $T_{c}=38 \mathrm{~K}$ and $W_{2}=$ $8.3 \mathrm{MeV}$ to solve for the $\lambda \mathrm{s}$. We remark that if among the four pairs of $\lambda$ values that are thus obtained there is a pair that automatically satisfies constraint (6), then (3) enables one to straightaway calculate two possible values for $W_{1}(0)$, one for each $\lambda$ together with the associated $\theta$. If one or both of these are in accord with experiment, then one is also led to the identification of the ion species responsible for it. This will happen only if the input values for the $T_{c}$ and $W_{2}(0)$ are accurately known. Generally, the latter of these is known with greater uncertainty, an uncertainty that GBCSEs can help to resolve.

7) Returning to the $\mathrm{Ba}+\mathrm{Fe}$ scenario under consideration, among the four pairs of $\lambda$ values that are obtained (Table 1), the pair corresponding to Debye temperatures $(1,3)$ is in gross disagreement with criterion $(6)$. Of the remaining three, all of which also violate (6), let us first consider the one that is closest to satisfying it:

$$
\lambda_{\mathrm{Ba}}^{c}=1.257, \lambda_{\mathrm{Fe}}^{c}=-0.271 .
$$

Now if $T_{c}$ is accurately known, then vary the value of $W_{2}(0)$ gradually till both the $\lambda$ s satisfy (6). Once this is achieved, calculate $\Delta_{1}(0)$ via (3)-with $\left(\lambda_{\mathrm{Ba}}^{c}, \theta_{\mathrm{Ba}}^{c}\right)$ and $\left(\lambda_{\mathrm{Fe}}^{c}, \theta_{\mathrm{Fe}}^{c}\right)$. Following this procedure we find that $W_{2}=$ $7.3 \mathrm{MeV}$ leads to $W_{\mathrm{Ba}}=3.05 \mathrm{MeV}$ (the experimental value is $\Delta_{1}(0)=3.6(0.5) \mathrm{MeV}$ [7] and $W_{2}(0)_{\mathrm{Fe}}=0.04$ $\mathrm{MeV}$ - via $\lambda$ s given in Table 1. Pertinent questions at this point are: why stop after a pair of $\lambda$ values satisfying constraint (6) is found? Why not seek to find even lower $\lambda$ values? One can do so, of course. However, this leads 
one farther away from the starting point of $W_{2}(0)=8.3$ $\mathrm{MeV}$. If $W_{2}(0)$ is believed to be accurately known, then apply the same procedure by varying $T_{c}$. In this case $T_{c}$ has to be increased in order to obtain the $\lambda$ s that satisfy constraint (6). Thus, $T_{c}=43 \mathrm{~K}$ yields $\lambda_{\mathrm{Ba}}^{c}=0.429$, $\lambda_{\mathrm{Fe}}^{c}=0.203$, which lead to $\Delta_{1}(0)_{\mathrm{Ba}}=2.31 \mathrm{MeV}, \Delta_{1}(0)_{\mathrm{Fe}}=$ $0.5 \mathrm{MeV}$. Also given in Table 1 are the results of a similar exercise for all the other pairs of $\lambda$ values not only in the $\mathrm{Ba}+\mathrm{Fe}$ scenario, but also in the $\mathrm{Ba}+\mathrm{As}$ scenario. We are thus led to the results: a) In the $\mathrm{Ba}+\mathrm{Fe}$ scenario, the only combination of Debye temperatures that can account for the experimental features of $\mathrm{BaAs}$ is $(2,3)$ since this leads to set $\{3.05 \mathrm{MeV}, 7.3 \mathrm{MeV}, 38 \mathrm{~K}\}$, the experimental range of the values of the gaps being: $\Delta_{1}(0)$ $=3.6(0.5) \mathrm{MeV}$ and $\Delta_{2}(0)=8.3(0.9) \mathrm{MeV}$; b) Similarly, in the $\mathrm{Ba}+\mathrm{As}$ scenario the only acceptable combination of Debye temperatures is $(2,6)$ which leads to the set $\{3.27 \mathrm{MeV}, 7.6 \mathrm{MeV}, 38 \mathrm{~K}$; c) In both cases the smaller gap is due to $\mathrm{Ba}$ and the theory has led to it per se; d) It seems interesting to note that if both the $\mathrm{Ba}+\mathrm{Fe}$ and the $\mathrm{Ba}+$ As scenarios are invoked for the formation of CPs then, in principle, one can account for the four gaps that have recently been reported from an ARPES experiment [11].

8) We now deal with approach a), paragraph c). Assuming that $\Delta_{1}(0)=3.6 \mathrm{MeV}$ and $\Delta_{2}(0)=8.3 \mathrm{MeV}$ [7], we seek to calculate the $T_{c}$ of the CS via (4) "after the $\lambda \mathrm{s}$ are determined via (3) and (5) by invoking both the $(\mathrm{Ba}$, $\mathrm{Fe})$ and the (Ba, As) pairs. The combinations of Debye temperatures that we now have to deal with, together with the results that they lead to, have been given in Table 2. This table also provides a guide, should it be needed, about employing approach b), paragraph c). The results in this table are not conclusive because of the uncertainties in both - the input values of $\Delta_{1}(0)$ and $\Delta_{2}(0)$. Nevertheless, it seems significant that the lowest best result for $T_{c}$, i.e. $40.7 \mathrm{~K}$, should have been led to by the $(2,6)$ combination of Debye temperatures as was the case above.

\subsection{Three-Gap Scenario}

The equality between $|W|$ (which is defined via the relation $2 E_{F}+W$ for the total energy of a CP where $E_{F}$ is the Fermi energy) and $\Delta[5]$ implies that there must be three $|W| \mathrm{s}$ if there are three gaps. We now take into account CPs bound via three-phonon exchanges in addition to those that are bound via one- and two-phonon exchanges. Equations (3) and (5) are therefore supplemented [14,15] by

$$
\begin{aligned}
1= & \lambda_{1}^{c} \ln \left[1+\frac{2 \hbar\left(\omega_{D}\right)_{1}^{c}}{\left|W_{3}(0)\right|}\right]+\lambda_{2}^{c} \ln \left[1+\frac{2 \hbar\left(\omega_{D}\right)_{2}^{c}}{\left|W_{3}(0)\right|}\right] \\
& +\lambda_{3}^{c} \ln \left[1+\frac{2 \hbar\left(\omega_{D}\right)_{3}^{c}}{\left|W_{3}(0)\right|}\right]
\end{aligned}
$$

Further, (4) now goes over to

$$
\begin{aligned}
1= & \lambda_{1}^{c} \int_{0}^{\theta_{1}^{c} / 2 T_{c}} \frac{\tanh (x)}{x} \mathrm{~d} x+\lambda_{2}^{c} \int_{0}^{\theta_{2}^{c} / 2 T_{c}} \frac{\tanh (x)}{x} \mathrm{~d} x \\
& +\lambda_{3}^{c} \int_{0}^{\theta_{3}^{c} / 2 T_{c}} \frac{\tanh (x)}{x} \mathrm{~d} x
\end{aligned}
$$

\begin{tabular}{|c|c|c|c|c|c|c|c|}
\hline \multicolumn{4}{|c|}{$\mathrm{Ba}+\mathrm{Fe}$ scenario } & \multicolumn{4}{|c|}{$\mathrm{Ba}+\mathrm{As}$ scenario } \\
\hline \multirow{2}{*}{$\begin{array}{c}\text { Debye temps } \\
(1,3)\end{array}$} & \multicolumn{2}{|c|}{$\lambda_{\mathrm{Ba}}, \lambda_{\mathrm{Fe}}$} & \multirow{2}{*}{$\begin{array}{c}T_{c} \\
47.9\end{array}$} & \multirow{2}{*}{$\frac{\text { Debye temps }}{(1,5)}$} & \multicolumn{2}{|c|}{$\lambda_{\mathrm{Ba}}, \lambda_{\mathrm{As}}$} & \multirow{2}{*}{$\begin{array}{c}T_{c} \\
47.8\end{array}$} \\
\hline & 0.355 & 0.121 & & & 0.355 & 0.124 & \\
\hline$(1,4)$ & 0.355 & 0.177 & 46 & $(1,6)$ & 0.355 & 0.192 & 45.5 \\
\hline$(2,3)$ & 0.515 & 0.153 & 42.2 & $(2,5)$ & 0.515 & 0.157 & 42.2 \\
\hline$(2,4)$ & 0.515 & 0.224 & 41.1 & $(2,6)$ & 0.515 & 0.242 & 40.7 \\
\hline$(3,1)$ & 0.333 & 0.125 & 48.4 & $(5,1)$ & 0.339 & 0.127 & 48.2 \\
\hline$(3,2)$ & 0.333 & 0.201 & 45.6 & $(5,2)$ & 0.339 & 0.204 & 45.4 \\
\hline$(4,1)$ & 0.449 & 0.154 & 44.1 & $(6,1)$ & 0.477 & 0.159 & 43.2 \\
\hline$(4,2)$ & 0.449 & 0.242 & 42 & $(6,2)$ & 0.477 & 0.256 & 41.2 \\
\hline
\end{tabular}

Table 2. Values of $\lambda$ s calculated via Equations (3) and (5) for different pairs of Debye temperatures given in Table 1, and the $T_{c^{-}}$-values that they lead to via Equation (4). With the exception of two of these which are only marginally greater than 0.5 (e.g., 0.515 in both the $\mathrm{Ba}+\mathrm{Fe}$ and the $\mathrm{Ba}+\mathrm{As}$ scenarios), all the $\lambda$ s satisfy constraint (6).

Notes on tables: Atomic masses used in the calculation of Debye temperatures are: 137.33 K (Ba), 39.098 K (K), $74.92 \mathrm{~K}(\mathrm{As}), 55.847 \mathrm{~K}$ (Fe). ${ }^{*}$ Entry marked (1) is obtained when $\mathrm{Ba}$ is the upper bob (and $\mathrm{K}$ the lower one; $\theta_{K}^{c}$ are not given as they are not used as shown) in the double pendulum; the one marked (2) is obtained with bobs interchanged. ${ }^{*}$ The $\theta_{i}^{c}$ in next two columns are to be similarly interpreted. *Note, however, that a pair of $\theta_{i}$ s that satisfies Equation (1) is (3) and (6), not (3) and (5). 


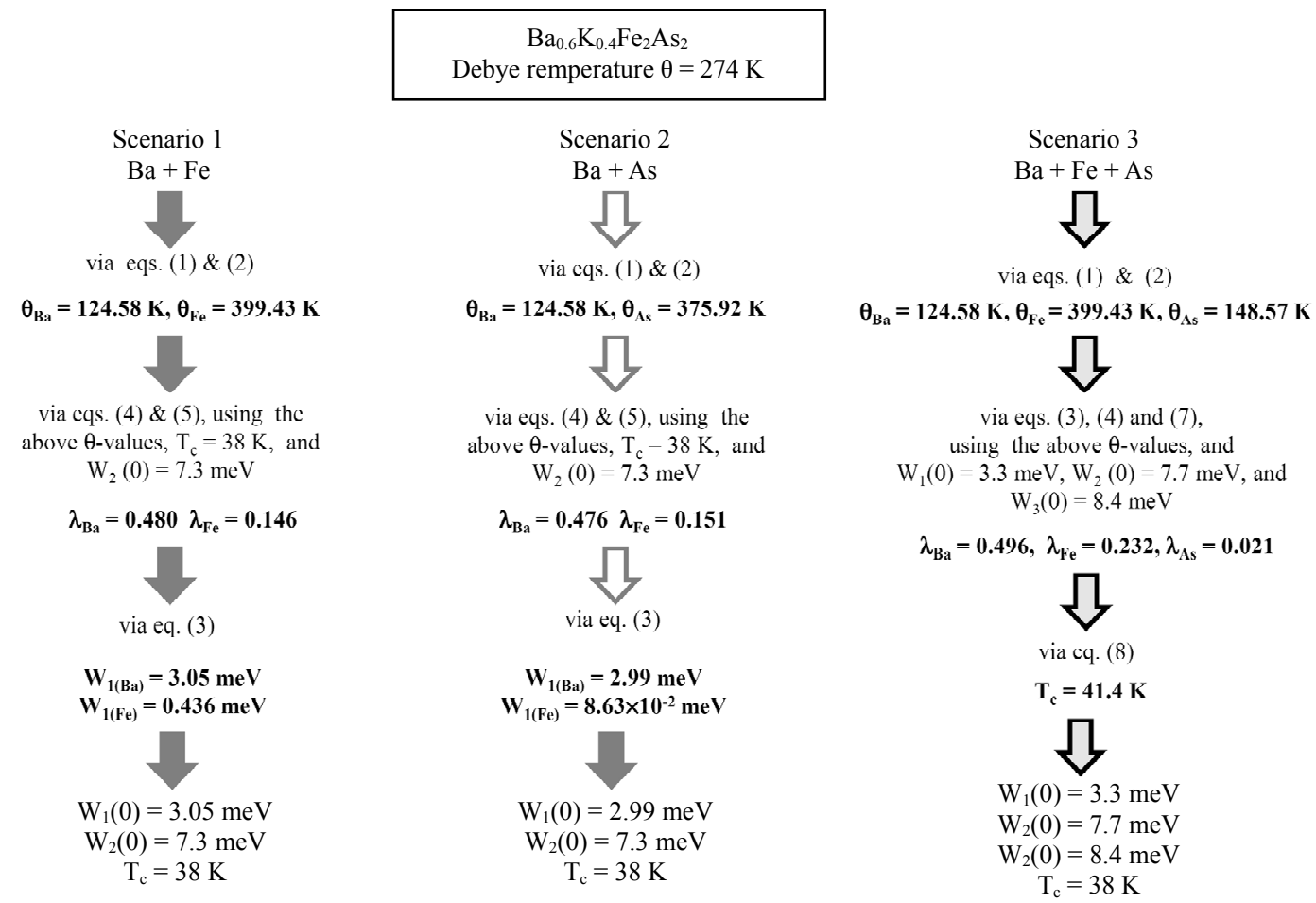

Figure 1. Schematic diagram giving the best-case values for the $T_{c}$ and the gap-values led to by $\mathrm{GBCSEs}_{\mathrm{for}} \mathrm{Ba}_{0.6} \mathrm{~K}_{0.4} \mathrm{Fe}_{2} \mathrm{As} \mathbf{s}_{2}$ based on its characterization via 2- and 3-gap scenarios.

Thus we have four Equations (3), (5), (7) and (8), involving the three interaction constants $\lambda_{1}^{c}, \lambda_{2}^{c}$ and $\lambda_{3}^{c}$ and which can be determined with the input of any three quantities from the set $\left\{\Delta_{1}(0), \Delta_{2}(0), \Delta_{3}(0), T_{c}\right\}$. Since the greatest uncertainty among these pertains to $\Delta_{3}(0)$, we first seek to calculate it by determining the three $\lambda$ s via (3), (5) and (8). Guided by the findings of Section 4.1, we invoke Debye temperature (2), see Table 1, for pairs bound via one-phonon exchanges, the combination of Debye temperatures $(2,6)$ for pairs bound via two-phonon exchanges, and the combination $(2,6,3)$ in the equation for $T_{c}$.

As our starting point for the inputs, we use the set: $\left\{\Delta_{1}(0)=3.6 \mathrm{MeV}, \Delta_{2}(0)=8.3 \mathrm{MeV}, 38 \mathrm{~K}\right\}$. This leads to values of the three $\lambda \mathrm{s}$ as $\{0.515,0.243,-0.02\}$. Since these values are in conflict with constraint (6), we first vary $\Delta_{1}(0)$ : lowering its value to $3.3 \mathrm{MeV}$ reduces the first two values to 0.496 and 0.26 , but the third value $(-0.02)$ remains unchanged. Keeping $\Delta_{1}(0)=3.3 \mathrm{MeV}$, we now reduce the value of $\Delta_{2}(0)$ whence, at $7.6 \mathrm{MeV}$, we find $\lambda_{2}^{c}=0.227$ and $\lambda_{3}^{c}=2.871 \times 10^{-5}$ while $\lambda_{1}^{c}$ remains unchanged at 0.496 . This set of $\lambda$ s leads to $\left|\Delta_{3}(0)\right|=7.6 \mathrm{MeV}$ via (7), which is not surprising because $\lambda_{3}^{c}$ is negligible. In a heuristic spirit, if we keep $\Delta_{1}(0)$ and $\Delta_{2}(0)$ fixed at 3.3 and $7.3 \mathrm{MeV}$ respectively, and increase $T_{c}$ from 38 to $42 \mathrm{~K},\left|\Delta_{3}(0)\right|$ attains a value of 8.5 $\mathrm{MeV}$.

As further illustrations of how the GBCSEs may be used, we draw attention to the gap values determined via the specific heat data [10]: 3.6, 8.5, and $9.2 \mathrm{MeV}$. One can determine the three $\lambda$ s by using these as input into (3), (5) and (7), and then calculate $T_{c}$ via (8). Using the (2, 6, 3 ) combination of temperatures, the values of $\lambda$ s so found are: $0.515,0.232$ and 0.021 . Since the first of these violates constraint (6), we need to vary $\Delta_{1}(0)$; changing its value from 3.6 to $3.3 \mathrm{MeV}$ without changing the values of $\Delta_{2}(0)$ and $\Delta_{3}(0)$, we find the $\lambda$-values to be: $0.496,0.27$ and 0.02 . Equation (8) now yields $T_{c}=44.5 \mathrm{~K}$. We note that the input of $3.3,7.7$, and $8.4 \mathrm{MeV}$ for the gap values leads to $0.496,0.232$, and 0.021 for the three $\lambda \mathrm{s}$, which lead to $T_{c}=41.4 \mathrm{~K}$. We finally note that, of course, one can also seek to determine $\Delta_{1}(0)$ or $\Delta_{2}(0)$ with the input of $\left\{\Delta_{2}(0)\right.$ or $\left.\Delta_{1}(0), \Delta_{3}(0), T_{c}\right\}$. These lead to results similar to those already quoted. The best scenarios are illustrated in Figure 1.

As just shown, two gaps and the $T_{c}$ of the iron-pnictide CS dealt with here are calculated by determining the sets $\left\{\lambda_{1}, \theta_{1}\right\}$ and $\left\{\lambda_{2}, \theta_{2}\right\}$. The use of both of these in (5) yields the larger gap, $\Delta_{2}$, while use of one of these in (3) yields the smaller gap, $\Delta_{1}$. Hence the question: what about the gap yielded by the other set via (3)? Not only in the present instance (see Table 1) but quite generally, the value of this gap is much smaller than $\Delta_{1}$ and often approaches zero $[14,15]$. Such gaps show up as nodes or lines of nodes on the Fermi surface and while for Ba-As they were reported by, among others [8], and have of late 
been studied with avid interest as evidenced by [17-19]. Note also that we found above a value of $2.871 \times 10^{-5}$ for $\lambda_{3}$ in the three-gap scenario, which leads to a vanishing value for gap. Based on [14,15] and the present work, we conjecture that the almost-vanishing gaps, such as that for $\lambda_{3}$, should be a feature of most (if not all) CSs. We note that this feature, as also reported in [17-19], appears to support the scenario in which CPs are formed via more than one phonon exchanges.

\section{Conclusions}

1) We first note that if the drop in the property (e.g., resistivity) of a CS is sharp, then there is no ambiguity in defining its $T_{c}$. If not, the mid-point of the temperaturerange over which the drop takes place is sometimes quoted as the $T_{c}$. The present study and also earlier work $[14,15]$ suggests that in applying GBCSEs, $T_{c}$ should be taken as the temperature which marks the onset of the said drop.

2) It was noted that the Debye temperature of the CS to which GBCSEs are applied is an important parameter. The value of this parameter used by us is the one quoted in [6] which need not necessarily be the value characterizing different samples of Ba-As for which the $T_{c}$ and the gap values have been quoted in the literature. The significance of the detailed quantitative results presented here needs to be assessed in light of this limitation.

3) Given the current experimental situation, we have shown that the GBCSEs can achieve for the iron pnictide $\mathrm{SC} \mathrm{Ba}-\mathrm{As}$, which is a CS, what the usual BCS equations do for simple SCs. Hence they may be of value in the ongoing work concerned with the other iron-pnictide SCs. Extension of our approach to deal with CSs that may be characterized by four gaps is straightforward. A tangible treatment for such an SC will, however, require precise experimental values of its $T_{c}$ and at least some of its gaps.

\section{Acknowledgements}

IC acknowledges support from CONACyT (Mexico) grant 103847, and MdeLl from UNAM-DGAPA-PAPIIT (Mexico) grant IN102011.

\section{REFERENCES}

[1] Y. Kamihara, T. Watanbe, M. Hirano and H. Hosono, "Iron-Based Layered Superconductor $\mathrm{La}\left[\mathrm{O}_{1-\mathrm{x}} \mathrm{Fe}_{\mathrm{x}}\right] \mathrm{FeAs}(\mathrm{x}$ $=0.05-0.12$ ) with $T_{c}=26 \mathrm{~K}$, , Journal of the American Chemical Society, Vol. 130, No. 11, 2008, pp. 3296-3297. doi:10.1021/ja800073m

[2] H. Takahashi, K. Igawa, K. Arii, Y. Kamihara, M. Hirano and $\mathrm{H}$. Hosono, "Superconductivity at $43 \mathrm{~K}$ in an IronBased Layered Compound $\mathrm{LaO}_{1-\mathrm{x}} \mathrm{F}_{\mathrm{x}} \mathrm{FeAs}$," Nature, Vol. 453, No. 7193, 2008, pp. 376-378. doi:10.10/nature06972

[3] K. Ishida, Y. Nakai and H. J. Hosono, "To What Extent
Iron-Pnictide New Superconductors Have Been Clarified: A Progress Report," Journal of the Physical Society of Japan, Vol. 78, 2009, Article ID: 062001. doi:10.1143/JPSJ.78.062001

[4] P. M. Aswathy, J. B. Anooja, P. M. Sarun and U. Syamaprasad, "An Overview on Iron Based Superconductors," Superconductor Science and Technology, Vol. 23, No. 7, 2010, Article ID: 073001. doi:10.1088/0953-2048/23/7/073001

[5] G. P. Malik, "On the Equivalence of Binding Energy of a Cooper Pair and the BCS Energy Gap: A Framework for Dealing with Composite Superconductors," International Journal of Modern Physics B, Vol. 24, No. 9, 2010, p. 1159. doi:10.1142/S0217979210055408

[6] G. Mu, H. Luo, Z. Wang, L. Shan, C. Ren and W. Wen, "Low Temperature Specific Heat of the Hole-Doped $\mathrm{Ba}_{0.6} \mathrm{~K}_{0.4} \mathrm{Fe}_{2} \mathrm{As}_{2}$ Single Crystals," Physical Review B, Vol. 79, No. 17, 2009, Article ID: 174501. doi:10.1103/PhysRevB.79.174501

[7] L. Shan, Y. Wang, B. Shen, B. Zeng, Y. Huang, A. Li, et al., "Observation of Ordered Vortices with Andreev Bound States in $\mathrm{Ba}_{0.6} \mathrm{~K}_{0.4} \mathrm{Fe}_{2} \mathrm{As}_{2}$," Nature Physics, Vol. 7, 2011, pp. 325-331. doi:10.1038/nphys1908

[8] H. Ding, P. Richard, K. Nakayama, K. Sugawara, T. Arakane, Y. Sekiba, et al., "Observation of Fermi-SurfaceDependent Nodeless Superconducting Gaps in

$\mathrm{Ba}_{0.6} \mathrm{~K}_{0.4} \mathrm{Fe}_{2} \mathrm{As}_{2}$," Europhysics Letters, Vol. 83, No. 4, 2008, Article ID: 47001. doi:10.1209/0295-5075/83/47001

[9] C. Ren, Z. Wang, H. Luo, H. Yang, L. Shan and W. Hai$\mathrm{Hu}$, "Evidence for Two Energy Gaps in Superconducting $\mathrm{Ba}_{0.6} \mathrm{~K}_{0.4} \mathrm{Fe}_{2} \mathrm{As}_{2}$ Single Crystals and the Breakdown of the Uemura Plot," Physical Review Letters, Vol. 101, No. 25, 2008, Article ID: 257006. doi:10.1103/PhysRevLett.101.257006

[10] P. Popovich, A. V. Boris, O. V. Dolgov, A. A. Golubov, D. L. Sun, C. T. Lin, et al., "Specific Heat Measurements of $\mathrm{Ba}_{0.6} \mathrm{~K}_{0.4} \mathrm{Fe}_{2} \mathrm{As}_{2}$ Single Crystals: Evidence for a Multiband Strong-Coupling Superconducting State," Physical Review Letters, Vol. 105, No. 2, 2010, Article ID: 027003. doi:10.1103/PhysRevLett.105.027003

[11] Y. Zhang, L. X. Yang, F. Chen, B. Zhou, X. F. Wang, X. H. Chen, X. H., et al., "Out-of-Plane Momentum and Symmetry-Dependent Energy Gap of the Pnictide

$\mathrm{Ba}_{0.6} \mathrm{~K}_{0.4} \mathrm{Fe}_{2} \mathrm{As}_{2}$ Superconductor Revealed by Angle-Resolved Photoemission Spectroscopy," Physical Review Letters, Vol. 105, No. 11, 2010, Article ID: 117003. doi:10.1103/PhysRevLett.105.027003

[12] F. Seitz, "The Modern Theory of Solids," McGraw Hill, New York, 1940.

[13] G. P. Malik and U. Malik, "On the Calculation of $T_{c}$ 's of Superconducting Binary Alloys Based on Two-Phonon Exchange Mechanism," Physica B, Vol. 348, No. 1-4, 2004, pp. 341-346. doi:10.1016/j.physb.2003.12.009

[14] G. P. Malik, "Generalized BCS Equations: Applications," Internantional Journal of Modern Physics B, Vol. 24, No. 19, 2010, p. 3701. doi:10.1142/S0217979210055858

[15] G. P. Malik and U. Malik, "A Study of the Thallium- and Bismuth-Based High-Temperature Superconductors in the 
Framework of the Generalized BCS Equations," Journal of Superconductivity and Novel Magnetism, Vol. 24, No. 1-2, 2011, pp. 255-260. doi:10.1007/s10948-010-1009-0

[16] J. M. Blatt, "Theory of Superconductivity," Academic Press, New York, 1964, p. 206.

[17] D. Lee, "Iron-Based Superconductors: Nodal Rings," Nature Physics, Vol. 8, 2012, pp. 364-365. doi:10.1038/nphys2301

[18] Y. Zhang, Z. R. Ye, Q. Q. Ge, F. Chen, J. Jiang, M. Xu, B.
P. Xie, et al., "Nodal Superconducting-Gap Structure in Ferropnictide Superconductor $\mathrm{BaFe}_{2}\left(\mathrm{As}_{0.7} \mathrm{P}_{0.3}\right)_{2}$," Nature Physics, Vol. 8, 2012, pp. 371-375. doi: $10.1038 /$ nphys 2248

[19] M. P. Allan, A. W. Rost, A. P. Mackenzie, Y. Xie, J. C. Davis, K. Klhou, et al., "Anisotropic Energy Gaps of Iron-Based Superconductivity from Intraband Quasiparticle Interference in LiFeAs," Science, Vol. 336, No. 6081, 2012, pp. 563-567. doi:10.1126/science. 1218726 